(C) [2008] IEEE. Reprinted, with permission, from [Silas Wan and Hung T. Nguyen, Human computer interaction using hand gesture, Engineering in Medicine and Biology Society, 2008. EMBS 2008. 30th Annual International Conference of the IEEE 2008]. This material is posted here with permission of the IEEE. Such ermission of the IEEE does not in any way imply IEEE endorsement of any of the University of Technology, Sydney's products or services. Internal or personal use of this material is permitted. However, permission to reprint/republish this material for advertising or promotional purposes or for creating new collective works for resale or redistribution must be obtained from the IEEE by writing to pubs-permissions@ieee.org. By choosing to view this document, you agree to all provisions of the copyright laws protecting it 


\title{
Human Computer Interaction using Hand Gesture
}

\author{
Silas Wan and Hung T. Nguyen, Senior Member, IEEE
}

\begin{abstract}
Hand gesture is a very natural form of human interaction and can be used effectively in human computer interaction (HCI). This project involves the design and implementation of a HCI using a small hand-worn wireless module with a 3-axis accelerometer as the motion sensor. The small stand-alone unit contains an accelerometer and a wireless Zigbee transceiver with microcontroller. To minimize intrusiveness to the user, the module is designed to be small ( $3 \mathrm{~cm}$ by $4 \mathrm{~cm}$ ). A time-delay neural network algorithm is developed to analyze the time series data from the 3-axis accelerometer. Power consumption is reduced by the noncontinuous transmission of data and the use of low-power components, efficient algorithm and sleep mode between sampling for the wireless module. A home control interface is designed so that the user can control home appliances by moving through menus. The results demonstrate the feasibility of controlling home appliances using hand gestures and would present an opportunity for a section of the aging population and disabled people to lead a more independent life.
\end{abstract}

\section{INTRODUCTION}

$\mathrm{T}$ HIS paper describes the design and implementation of an accelerator-based hand gesture classification system which can be used for human computer interaction (HCI) applications. A home control interface is designed so that the user can control home appliances by moving through menus. This application is targeted towards the aged and disabled people who have problems using remote control.

The main approaches for analyzing and classifying hand gestures for HCI include glove-based techniques and visionbased techniques. The glove-based techniques use sensors to measure the positions of the fingers and the position of the hand in real-time [1-4]. The Nintendo Power Glove, launched in 1989, was the first peripheral that created human hand gestures on the television or computer screen. It uses bend sensors to detect movement of fingers as well as magnetic/inertia tracking devices to track the pitch, yaw, roll and acceleration of the whole glove. The CyberGlove II from Immersion is a more sophisticated system which has multiple sensors such as bend sensors, abduction sensors and palm-arc sensors and can be used to obtain accurate three-dimensional representations of the hand. Recently, it was used to describe ambiguous sets of phrases consisting of three to five hand gestures [1]. However, gloves tend to be

This work was supported in part by Australian Research Council under Discovery Grant DP0666942.

H. T. Nguyen is with Faculty of Engineering, University of Technology, Sydney, Broadway, NSW 2007, Australia (phone: +612-9514-2451; fax: +61 29514 2868; e-mail: Hung.Nguyen@uts.edu.au).

S. Wan is with Faculty of Engineering, University of Technology Sydney, Broadway, NSW 2007, Australia (e-mail: silas.wan@student.uts.edu.au). expensive and in general do not promote free movement of the hand.

The vision-based techniques can be either 3D or 2D approaches. As 3D hand models are quite complex, the classification of gestures from 3D models is computationally intensive making real-time implementation difficult. On the other hand, the 2D approach is restricted to the less complex problem of classifying well-defined static gestures [5-8].

Recent existing systems using inertial devices include the Nintendo Wii. It uses the ADXL330 accelerometer from Analog Devices. The nunchuk uses the LIS3L02AL accelerometer from STMicroelectronics. The Wii remote is a wireless device that communicates with the Nintendo Wii via Bluetooth. To obtain absolute position for games, an infrared sensor is used (Wii Infrared Sensor Bar). Infrared sensors, unlike accelerometers, provide good low frequency information but not high frequency information. Thus the two sensors can be fused using optimal filtering to obtain accurate position and movement information.

In this paper, we describe the development of both hardware and software for a wireless hand gesture recognition system. Section II provides an overview of the hand gesture recognition module design and the relevant software for the system. Section III presents the development and results of an embedded neural network implementation. Section IV provides a conclusion for this study.

\section{METHODS}

\section{A. Wireless Hand Gesture Module}

The Sensor Node Block as shown in Fig. 1 is the heart of this project. It consists of a Jennic JN5139 wireless transceiver module, an Analog Devices ADXL330 accelerometer, a lithium polymer battery and a charger system. The Jennic JN5139 Zigbee Module is a surface mount module that allows Zigbee systems to be implemented with a short development time and low cost. It allows applications to be developed very quickly without going into details of RF antenna and oscillator design. All $\mathrm{RF}$ components are included in the module. It contains a Jennic JN5139 wireless microcontroller, ceramic antenna and a 1MBit flash for program memory. The flash memory can also be used for storage and in this project, it is used to store the parameters for the hand gesture recognition neural network. The Analog Devices ADXL330 is used in this module. It is a three axis accelerometer that provides $\pm 3 g$ range in a small $4 \mathrm{~mm} \times 4 \mathrm{~mm}$ package.

Power supply is a very critical to wireless sensors. The battery must be small and lightweight to be practical. Lithium polymer batteries are chosen because they are 
rechargeable in circuit, have higher voltage and higher capacity. The lithium polymer used are UBC581730 from UltraLife. The Microchip MCP73811 Integrated Charge Management Controller is the heart of the battery charging circuit. It is a 5 pin SOT23 surface mount IC that provides specific charge algorithms for single cell lithium polymer or lithium iron batteries to achieve optimal charging in the shortest possible time. An additional link is also made to the board design to allow the battery to be charged via the programming header, which gets its power from the USB port.

A six-way header is used to program the modules. The module can be programmed using the Jennic Codeblocks SDK in the C language. In the programming mode, the PC is connected to the header via a USB cable which contains a FTDI FT232 conversion IC in the cable connecting housing. The programming header also provides $5 \mathrm{~V}$ from the USB which is used to charge the Lithium Polymer battery.

\section{B. Software for the Hand Gesture Recognition System}

There are several important software features which have been implemented with the system. They include the dropout detection and re-connection of the wireless communication, as well as the battery voltage monitor module.

The Jennic JN5139 Zigbee wireless microcontroller contains a supply monitor function inside its Analog Peripheral module. The supply voltage is compared with the voltage regulator output of the wireless microcontroller through a voltage divider. As the supply voltage is not expected to change rapidly, the supply monitor is only activated once per second. The information is sent to PC interface so the user can continuously monitor the supply voltage on the PC screen. When the supply voltage falls below $2.97 \mathrm{~V}$, or $10 \%$ above the minimum operating voltage of the Jennic module of $2.7 \mathrm{~V}$, the PC interface will flash an alarm to the user.

One of the key features of this project is the ability of the system to adapt to different users through flexible reprogramming of neural network weights. Although the neural network architecture can be programmed into the modules, the weights have to be transferred from the PC, where the neural network is trained, to the modules.

In this project, we allow the weights to be programmed wirelessly. This method makes use of the $128 \mathrm{kB}$ serial Flash memory that comes with the Jennic modules. The flash memory used are either ST M25P10 (for evaluation kit) or SST 25VF010 (standalone module). Flash read/write functions for these two flash ICs are provided by Jennic and the functions are identical in its way of use. Each flash IC is divided into four sectors. Sectors 1, 2 and 3 contain the Zigbee protocol, MAC addresses and applications code. Sector 3 is free to use as storage and houses the neural network weights and look up table. The neural parameters are first trained in Matlab. The parameters are read in the user interface and then organized into packets to be sent to the wireless module.

The Zigbee protocol is used with this module. Unlike Bluetooth, Zigbee is designed for short range communication for intelligent devices with low data rate and low current consumption. Zigbee is also capable of forming mesh networks with other Zigbee devices while Bluetooth can only transmit one to one. Both protocols operate under the $2.4 \mathrm{GHz}$ ISM band which is free to use.

The Sensor Node contains a JN5139 module which uses a proprietary microcontroller from Jennic. For this project, the microcontroller has been programmed to contain the following features: acquire accelerometer reading, execute the embedded neural network algorithm, transmit results or raw acceleration data to the PC interface, and program neural network weights wirelessly. The main software routine of the Sensor Node is a timer callback function as shown in Figure 2. The other independent routine is the Zigbee packets from the Receiver Node which is shown in Figure 3. All control messages are received through Zigbee.

The Receiver Node mainly acts as a relay. It has three main independent software routines. The UART routine receives serial packets from the PC interface while the Zigbee routine receives Zigbee messages from the Sensor Node. The Receiver Node timer is used in Sensor Node drop out detection and to repeat sending Zigbee commands until the Sensor Node receives them.

A PC interface as shown in Fig. 4 is written in Borland $\mathrm{C}++$ Builder. It contains the following features: display and acceleration data, load neural network weights, execute realtime neural network algorithm, activate alarm when battery level is too low or the Sensor Node cannot be detected, and control the Sensor Node remotely.

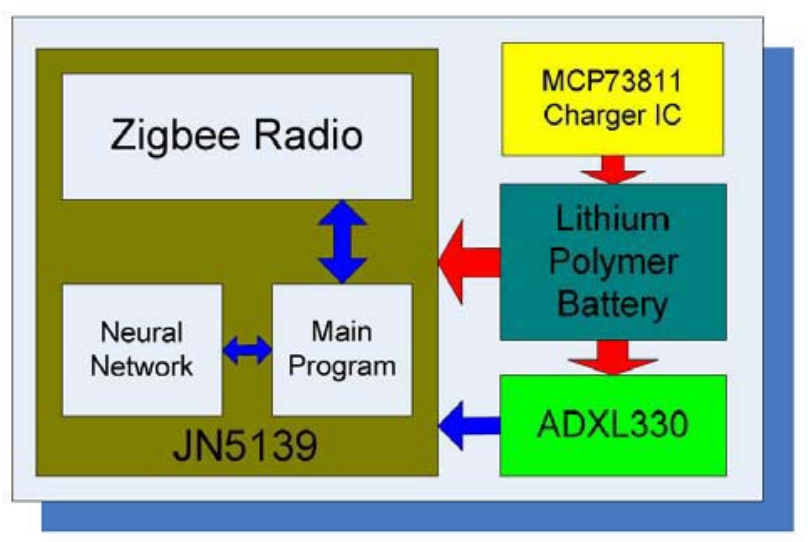

Fig. 1. Sensor Node Block Diagram 

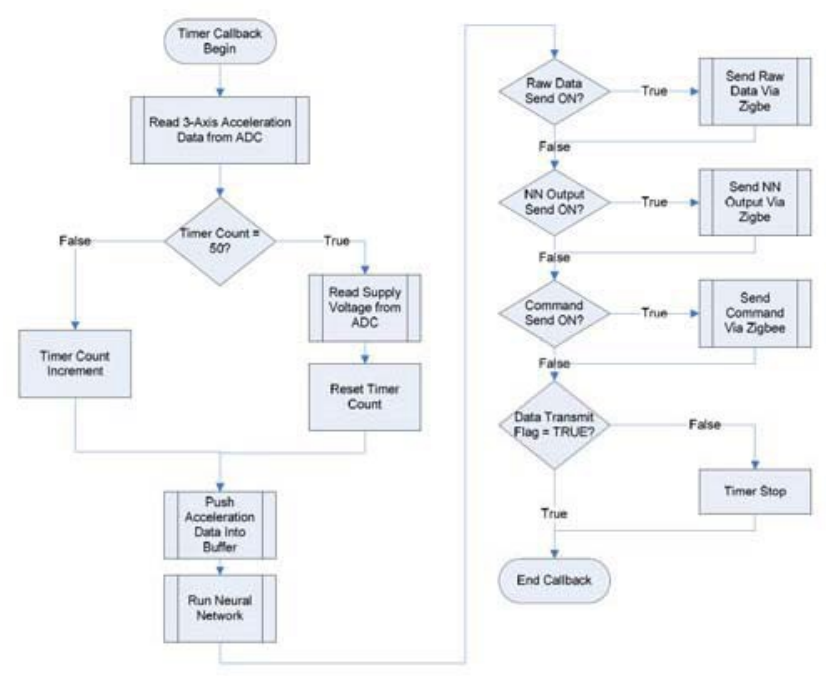

Fig. 2. Sensor Node Timer Callback Flowchart

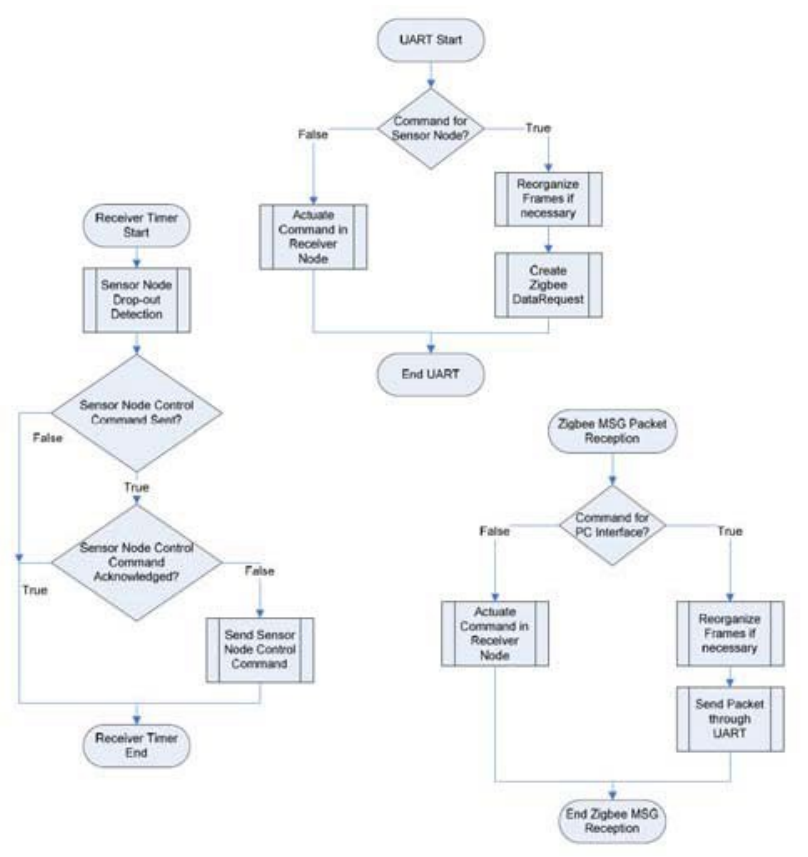

Fig. 3. Receiver Node Flowcharts

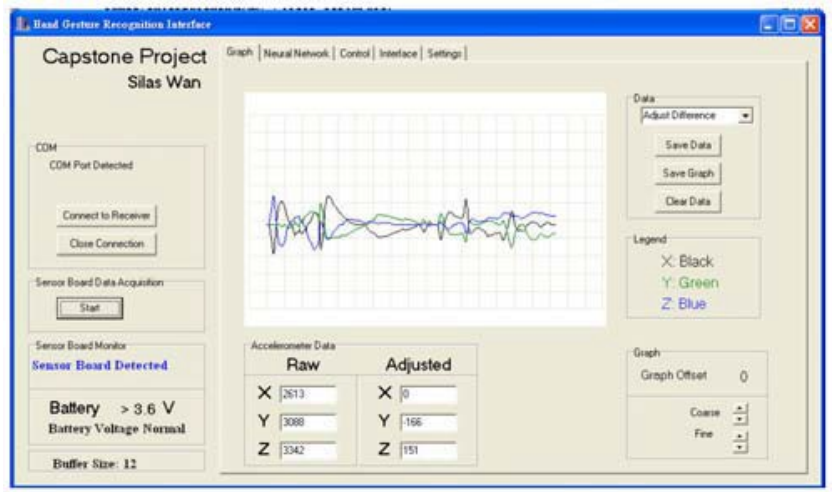

Fig. 4. Hand Gesture Recognition System PC Interface

\section{Home Control GUI}

The Home Control GUI as shown in Fig. 5 is a simple interface that allows the user to control basic home appliances. It illustrates how the technology could potentially be used by people in their homes. Many people in the aging population and disabled people have difficulty using a remote control as it requires sophisticated hand grasp and button movements. Hand gesture is much more natural and the system can be trained to recognize different hand gesture, even when they are extremely irregular and noisy. For severely disabled people who can not use their hands at all, we have also experimented with hands-free control systems using head movement or EEG-based devices [9-11].

\section{Neural Network Classifier}

The accelerometer outputs three vectors $\mathrm{a}_{\mathrm{x}}, \mathrm{a}_{\mathrm{y}}$ and $\mathrm{a}_{\mathrm{z}}$. These outputs are cascaded together as a string to form an input to the classifier neural network. We also use baseline adjustment to remove the average value from the windowed input. It also ensures that every individual hand movement have a zero crossing, since a hand movement involves both start and stop which have opposite directions for acceleration. Height normalization is also implemented to make the input data of identical hand movements look more similar. For embedded neural network implementation, the memory limitation of the Jennic modules means that the network weights cannot be stored as floating points. Instead, the weights are stored as integers. For this reason, a large amount of scaling, level shifting and thresholding is necessary to deal with floating points.

\section{RESULTS}

The Wireless Hand Gesture Module has been implemented as shown in Fig. 6. Its overall size is $3 \mathrm{~cm} \times$ $4 \mathrm{~cm} \times 1 \mathrm{~cm}$ and current consumption is $45 \mathrm{~mA}$. It takes about 45 minutes to charge the batteries. For a continuous drain of 50mA current, the batteries will last about 5 hours.

For the Home Control example, five movements were trained: up, left, down, right and click (OK). The accuracies of the five hand movements are shown in Table 1 . The percentage of success is $86.3 \%$. It is quite difficult to get a high success rate for hand gesture. However, when the user adapts to the necessary hand gesture, the success rate can be extremely high and approaches $100 \%$. This demonstrates the power of hand gesture - the user can adapt to what the computer expects.

Tests around the laboratory indicate that the device still functions if the Receiver and Sensor Nodes are on opposite side of the laboratory, at about $15 \mathrm{~m}$. 


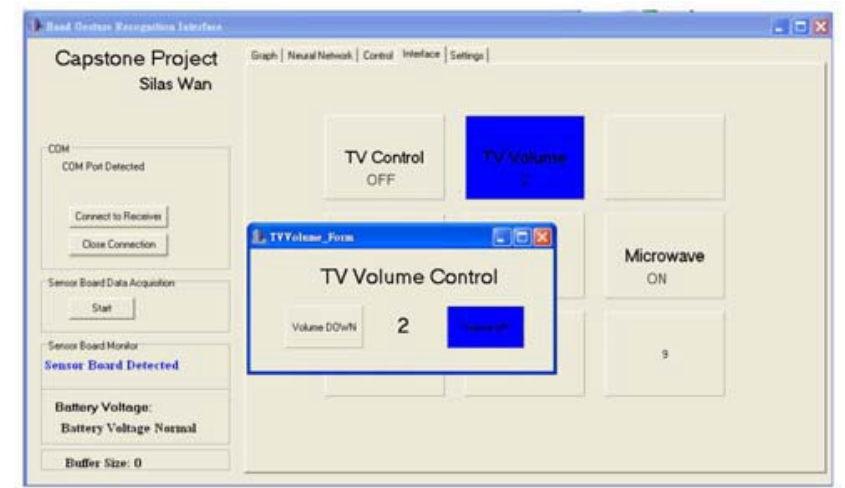

Fig. 5. Home Control GUI

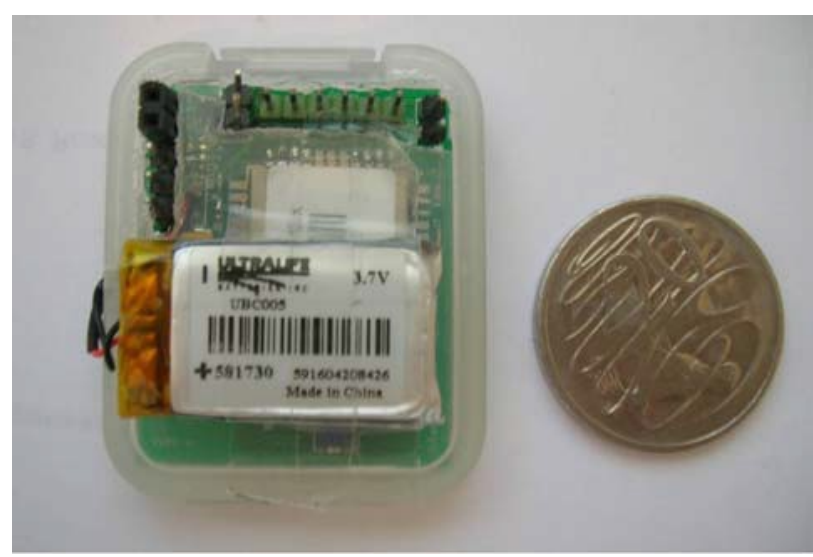

Fig. 6. Wireless Hand Gesture Module

TABLE I

HAND GESTURE RECOGNITION

\begin{tabular}{|c|c|c|}
\hline Command & Total & $\begin{array}{c}\text { Correctly } \\
\text { Recognized }\end{array}$ \\
\hline Up & 60 & 54 \\
\hline Left & 60 & 50 \\
\hline Down & 60 & 43 \\
\hline Right & 60 & 52 \\
\hline Click & 60 & 60 \\
\hline Total & 300 & 259 \\
\hline Percentage & $100 \%$ & $86.3 \%$ \\
\hline
\end{tabular}

The results indicate the project is a success overall. The hardware is working perfectly and able to be recharge through the USB port. The electronic noise level is sufficiently low to not interfere with the ceramic antenna of the Jennic module. Various other software features also successfully allow the user to interact with the hardware easily.

\section{CONCLUSION}

This project demonstrated the feasibility of designing and implementing hand gesture recognition devices using a three-axis accelerometer, a small wireless transceiver module as well as implementing an embedded neural network in the device to make it standalone. The Home Control Interface also demonstrates the feasibility of control home appliances using the hand gesture, and would present an opportunity for a section of the aging population and disabled people to lead a more independent life.

\section{REFERENCES}

[1] B. W. Miners, O.A. Basir, M. S. Kamel, "Understanding Hand Gestures using Approximate Graph Matching”, IEEE Trans. on Systems, Man, and Cybernetics - Part A: Systems and Humans, Vol. 35, No. 2, pp. 239-248, 2005.

[2] M-C Su, “A Fuzzy Rule-Based Approach to Spatio-Temporal Hand Gesture Recognition”, IEEE Trans. on Systems, Man, and Cybernetics - Part C: Applications and Reviews, Vol. 30, No.2, pp. 276-281, 2000.

[3] I. Dejmal, M. Zacksenhouse, "Coordinate Structure of Manipulative Hand-Movements Facilitates their Recognition", IEEE Trans. on Biomedical Engineering, Vol. 53, No. 12, pp. 2455-2463, 2006.

[4] Y. Nam, K. Wohn, L-K Hyung, "Modeling and Recognition of Hand Gesture using Colored Petri Net”, IEEE Trans. on Systems, Man, and Cybernetics - Part A: Systems and Humans, Vol. 29, No. 5, pp. 516-521, 1999.

[5] L. Gupta, S. Ma, “ Gesture-based Interaction and Communication: Automated Classification of Hand Gesture Contours”, IEEE Trans. on Systems, Man, and Cybernetics - Part C: Application and Reviews, Vol. 31, No. 1, pp. 114-120, 2001.

[6] V. I. Pavlovic, R. Sharma, T. S. Huang, "Visual Interpretation of Hand Gestures for Human-Computer Interaction: A Review”, IEEE Trans. on Pattern Analysis and Machine Intelligence, Vol. 19, No. 7, pp. 677-695, 1997.

[7] S. P. Kang, G. Rodnay, M. Tordan, Katupitiya, “A Hand Gesture Based Virtual Interface for Wheelchair Control “, Proceedings of the 2003 IEEE/ASME International Conference on Advanced Intelligent Mechatronics, pp. 77-783, 2003.

[8] H. Je, J. Kim, D. Kim, "Vision-based Hand Gesture Recognition for understanding Musical Time Pattern and Tempo", 33 ${ }^{\text {rd }}$ Annual Conferences of the IEEE Industrial Electronics Society, Taipei, Taiwan, November 5-9, pp. 2371-2376, 2007.

[9] H. T. Nguyen, S. T. Nguyen, P. B. Taylor, J. Middleton, "Head Direction Command Classification using an Adaptive Optimal Bayesian Neural Network". International Journal of Factory Automation, Robotics and Soft Computing, No. 3, pp. 98-103, 2007.

[10] H. T. Nguyen, L. M. King, G. Knight, "Real-time Head Movement System and Embedded Linux Implementation for the Control of Power Wheelchairs", 26 ${ }^{\text {th }}$ Annual International Conference of the IEEE Engineering in Medicine and Biology Society, San Francisco, USA, September 1-5, pp. 4892-4895, 2004.

[11] D. A. Craig, H. T. Nguyen, H. A. Burchey, "Control of Power Wheelchair using EEG-based Thought Pattern Recognition”, $28^{\text {th }}$ Annual International Conference of the IEEE Engineering in Medicine and Biology Society, New York City, USA, August 31September 3, pp. 1291-1294, 2006 